\title{
Furstenberg transformations on Cartesian products of infinite-dimensional tori
}

\author{
P. A. Cecchi ${ }^{1}$ and R. Tiedra de Aldecoa ${ }^{2 *}$ \\ 1 Departamento de Matemáticas, Universidad de Santiago de Chile, \\ Av. Alameda Libertador Bernardo O'Higgins 3363, Estación Central, Santiago, Chile \\ 2 Facultad de Matemáticas, Pontificia Universidad Católica de Chile, \\ Av. Vicuña Mackenna 4860, Santiago, Chile \\ E-mails: pcecchib@gmail.com, rtiedra@mat.puc.cl
}

\begin{abstract}
We consider in this note Furstenberg transformations on Cartesian products of infinite-dimensional tori. Under some appropriate assumptions, we show that these transformations are uniquely ergodic with respect to the Haar measure and have countable Lebesgue spectrum in a suitable subspace. These results generalise to the infinite-dimensional setting previous results of $H$. Furstenberg, A. Iwanik, M. Lemańzyk, D. Rudolph and the second author in the one-dimensional setting. Our proofs rely on the use of commutator methods for unitary operators and Bruhat functions on the infinite-dimensional torus.
\end{abstract}

2010 Mathematics Subject Classification: 28D10, 37A30, 37C40, 58J51.

Keywords: Furstenberg transformations, infinite-dimensional torus, commutator methods.

\section{Introduction}

We consider in this note the generalisation of Furstenberg transformations on Cartesian products $\mathbb{T}^{d}$ $(d \geq 2)$ of one-dimensional tori [8, Sec. 2] to the case of Cartesian products $\left(\mathbb{T}^{\infty}\right)^{d}$ of infinite-dimensional tori. Using recent results on commutator methods for unitary operators $[14,15]$, we show under a $C^{1}$ regularity assumption on the perturbations that these transformations are uniquely ergodic with respect to the Haar measure on $\left(\mathbb{T}^{\infty}\right)^{d}$ and are strongly mixing in the orthocomplement of functions depending only on variables in the first torus $\mathbb{T}^{\infty}$ (see Assumption 3.2 and Theorem 3.3). Under a slightly stronger regularity assumption ( $C^{1}+$ Dini continuous derivative), we also show that these transformations have countable Lebesgue spectrum in that orthocomplement (see Theorem 3.4). These results generalise to the infinite-dimensional setting previous results of H. Furstenberg, A. Iwanik, M. Lemańzyk, D. Rudolph and the second author $[8,10,15]$ in the one-dimensional setting.

Apart from commutator methods, our proofs rely on the use of Bruhat test functions [5] which provide a natural analog to the usual $C^{\infty}$-functions which do not exist in our infinite-dimensional setting (see Section 3 for details). We also mention that all the results of this note apply to Furstenberg transformations on Cartesian products $\left(\mathbb{T}^{n}\right)^{d}$ of finite-dimensional tori $\mathbb{T}^{n}$ of any dimension $n \geq 1$. One just has to consider the particular case where the functions defining the Furstenberg transformations on $\left(\mathbb{T}^{\infty}\right)^{d}$ depend only on a finite number of variables.

\footnotetext{
*Supported by the Chilean Fondecyt Grant 1130168 and by the Iniciativa Cientifica Milenio ICM RC120002 "Mathematical Physics" from the Chilean Ministry of Economy.
} 
Here is a brief description of the content of the note. First, we recall in Section 2 the needed facts on commutators of unitary operators and regularity classes associated to them. Then, we define in Section 3 the Furstenberg transformations on $\left(\mathbb{T}^{\infty}\right)^{d}$, and prove Theorems 3.3 and 3.4 on the unique ergodicity, strong mixing property and countable Lebesgue spectrum of these transformations.

We refer to $[9,11,12,13]$ and references therein for other works building on Furstenberg transformations.

Acknowledgements. The authors thank M. Măntoiu and J. Rivera-Letelier for discussions which partly motivated this note.

\section{Commutator methods for unitary operators}

We recall in this section some facts borrowed from $[7,14]$ on commutator methods for unitary operators and regularity classes associated to them.

Let $\mathcal{H}$ be a Hilbert space with scalar product $\langle\cdot, \cdot\rangle$ antilinear in the first argument, denote by $\mathscr{B}(\mathcal{H})$ the set of bounded linear operators on $\mathcal{H}$, and write $\|\cdot\|$ both for the norm on $\mathcal{H}$ and the norm on $\mathscr{B}(\mathcal{H})$. Let $A$ be a self-adjoint operator in $\mathcal{H}$ with domain $\mathcal{D}(A)$, and take $S \in \mathscr{B}(\mathcal{H})$. For any $k \in \mathbb{N}$, we say that $S$ belongs to $C^{k}(A)$, with notation $S \in C^{k}(A)$, if the map

$$
\mathbb{R} \ni t \mapsto \mathrm{e}^{-i t A} S \mathrm{e}^{i t A} \in \mathscr{B}(\mathcal{H})
$$

is strongly of class $C^{k}$. In the case $k=1$, one has $S \in C^{1}(A)$ if and only if the quadratic form

$$
\mathcal{D}(A) \ni \varphi \mapsto\langle\varphi, S A \varphi\rangle-\langle A \varphi, S \varphi\rangle \in \mathbb{C}
$$

is continuous for the topology induced by $\mathcal{H}$ on $\mathcal{D}(A)$. We denote by $[S, A]$ the bounded operator associated with the continuous extension of this form, or equivalently $-i$ times the strong derivative of the function (2.1) at $t=0$.

A condition slightly stronger than the inclusion $S \in C^{1}(A)$ is provided by the following definition: $S$ belongs to $C^{1+0}(A)$, with notation $S \in C^{1+0}(A)$, if $S \in C^{1}(A)$ and if $[A, S]$ satisfies the Dini-type condition

$$
\int_{0}^{1} \frac{\mathrm{d} t}{t}\left\|\mathrm{e}^{-i t A}[A, S] \mathrm{e}^{i t A}-[A, S]\right\|<\infty
$$

As banachisable topological vector spaces, the sets $C^{2}(A), C^{1+0}(A), C^{1}(A)$ and $C^{0}(A) \equiv \mathscr{B}(\mathcal{H})$ satisfy the continuous inclusions [2, Sec. 5.2.4]

$$
C^{2}(A) \subset C^{1+0}(A) \subset C^{1}(A) \subset C^{0}(A) .
$$

Now, let $U \in C^{1}(A)$ be a unitary operator with (complex) spectral measure $E^{U}(\cdot)$ and spectrum $\sigma(U) \subset \mathbb{S}^{1}:=\{z \in \mathbb{C}|| z \mid=1\}$. If there exist a Borel set $\Theta \subset \mathbb{S}^{1}$, a number $a>0$ and a compact operator $K \in \mathscr{B}(\mathcal{H})$ such that

$$
E^{U}(\Theta) U^{*}[A, U] E^{U}(\Theta) \geq a E^{U}(\Theta)+K
$$

then one says that $U$ satisfies a Mourre estimate on $\Theta$ and that $A$ is a conjugate operator for $U$ on $\Theta$. Also, one says that $U$ satisfies a strict Mourre estimate on $\Theta$ if (2.2) holds with $K=0$. One of the consequences of a Mourre estimate is to imply spectral properties for $U$ on $\Theta$. We recall here these spectral results in the case $U \in C^{1+0}(A)$. We also recall a result on the strong mixing property of $U$ in the case $U \in C^{1}(A)$ (see [7, Thm. 2.7 \& Rem. 2.8] and [14, Thm. 3.1] for more details). 
Theorem 2.1 (Absolutely continuous spectrum). Let $U$ and $A$ be respectively a unitary and a self-ajoint operator in a Hilbert space $\mathcal{H}$, with $U \in C^{1+0}(A)$. Suppose there exist an open set $\Theta \subset \mathbb{S}^{1}$, a number a $>0$ and a compact operator $K \in \mathscr{B}(\mathcal{H})$ such that

$$
E^{U}(\Theta) U^{*}[A, U] E^{U}(\Theta) \geq a E^{U}(\Theta)+K
$$

Then, $U$ has at most finitely many eigenvalues in $\Theta$, each one of finite multiplicity, and $U$ has no singular continuous spectrum in $\Theta$. Furthermore, if (2.3) holds with $K=0$, then $U$ has only purely absolutely continuous spectrum in $\Theta$ (no singular spectrum).

Theorem 2.2 (Strong mixing). Let $U$ and $A$ be respectively a unitary and a self-adjoint operator in a Hilbert space $\mathcal{H}$, with $U \in C^{1}(A)$. Assume that the strong limit

$$
D:=s_{N \rightarrow \infty}-\lim _{\ell} \frac{1}{N} \sum_{\ell=0}^{N-1} U^{\ell}\left([A, U] U^{-1}\right) U^{-\ell}
$$

exists, and suppose that $\eta(D) \mathcal{D}(A) \subset \mathcal{D}(A)$ for each $\eta \in C_{c}^{\infty}(\mathbb{R} \backslash\{0\})$. Then,

(a) $\lim _{N \rightarrow \infty}\left\langle\varphi, U^{N} \psi\right\rangle=0$ for each $\varphi \in \operatorname{ker}(D)^{\perp}$ and $\psi \in \mathcal{H}$,

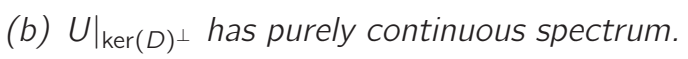

\section{Furstenberg transformations on Cartesian products of infinite- dimensional tori}

Let $\mathbb{T}^{\infty} \simeq \mathbb{R}^{\infty} / \mathbb{Z}^{\infty}$ be the infinite-dimensional torus with elements $x \equiv\left\{x_{k}\right\}_{k=1}^{\infty}$, and let $\widehat{\mathbb{T}^{\infty}}$ be the dual group of $\mathbb{T}^{\infty}$. For each $n \in \mathbb{N}_{\geq 1}$, let $\mu_{n}$ be the normalised Haar measure on $\left(\mathbb{T}^{\infty}\right)^{n}$, and let $\mathcal{H}_{n}:=$ $\mathrm{L}^{2}\left(\left(\mathbb{T}^{\infty}\right)^{n}, \mu_{n}\right)$ be the corresponding Hilbert space. In analogy to Furstenberg transformations on Cartesian products of one-dimensional tori (see [8, Sec. 2.3]), we consider Furstenberg transformations on Cartesian products of infinite-dimensional tori $T_{d}:\left(\mathbb{T}^{\infty}\right)^{d} \rightarrow\left(\mathbb{T}^{\infty}\right)^{d}, d \in \mathbb{N}_{\geq 2}$, given by

$$
T_{d}\left(x_{1}, x_{2}, \ldots, x_{d}\right):=\left(x_{1}+\alpha, x_{2}+\phi_{1}\left(x_{1}\right), \ldots, x_{d}+\phi_{d-1}\left(x_{1}, x_{2}, \ldots, x_{d-1}\right)\right),
$$

with $\alpha \in \mathbb{T}^{\infty}$ such that $\{n \alpha\}_{n \in \mathbb{Z}}$ is dense in $\mathbb{T}^{\infty}$ and $\phi_{j} \in C\left(\left(\mathbb{T}^{\infty}\right)^{j} ; \mathbb{T}^{\infty}\right)$ for each $j \in\{1, \ldots, d-1\}$. Since $T_{d}$ is invertible and preserves the measure $\mu_{d}$, the Koopman operator

$$
W_{d}: \mathcal{H}_{d} \rightarrow \mathcal{H}_{d}, \quad \varphi \mapsto \varphi \circ T_{d},
$$

is a unitary operator. Furthermore, $W_{d}$ is reduced by the orthogonal decompositions

$$
\mathcal{H}_{d}=\mathcal{H}_{1} \bigoplus_{j \in\{2, \ldots, d\}}\left(\mathcal{H}_{j} \ominus \mathcal{H}_{j-1}\right)=\mathcal{H}_{1} \bigoplus_{j \in\{2, \ldots, d\}, \chi \in \widehat{\mathbb{T} \infty} \backslash\{1\}} \mathcal{H}_{j, \chi}, \quad \mathcal{H}_{j, \chi}:=\left\{\eta \otimes \chi \mid \eta \in \mathcal{H}_{j-1}\right\},
$$

and the restriction $\left.W_{d}\right|_{\mathcal{H}_{j, x}}$ is unitarily equivalent to the unitary operator

$$
U_{j, \chi} \eta:=\left(\chi \circ \phi_{j-1}\right) W_{j-1} \eta, \quad \eta \in \mathcal{H}_{j-1} .
$$

In order to define later a conjugate operator for $U_{j, x}$, we first define a suitable group of translations on $\left(\mathbb{T}^{\infty}\right)^{j-1}$. For this, we choose $\left\{y_{t}\right\}_{t \in \mathbb{R}}$ an ergodic continuous one-parameter subgroup of $\mathbb{T}^{\infty}$ such that each map

$$
\mathbb{R} \ni t \mapsto\left(\left(y_{t}\right)_{1}, \ldots,\left(y_{t}\right)_{n}\right) \in \mathbb{T}^{n}, \quad n \in \mathbb{N}_{\geq 1},
$$

is of class $C^{1}$. An example of such a subgroup $\left\{y_{t}\right\}_{t \in \mathbb{R}}$ is for instance given by the formula

$$
\left(y_{t}\right)_{k}=y^{k} t \quad \bmod \mathbb{Z}, \quad t \in \mathbb{R}, \quad k \in \mathbb{N}_{\geq 1}
$$


with $y \in \mathbb{R}$ a transcendental number (see [6, Ex. 4.1.1]). Then, we associate to $\left\{y_{t}\right\}_{t \in \mathbb{R}}$ the translation flow

$$
F_{j-1, t}\left(x_{1}, \ldots, x_{j-1}\right):=\left(x_{1}, \ldots, x_{j-1}+y_{t}\right), \quad t \in \mathbb{R},\left(x_{1}, \ldots, x_{j-1}\right) \in\left(\mathbb{T}^{\infty}\right)^{j-1},
$$

and the translation operators $V_{j-1, t}: \mathcal{H}_{j-1} \rightarrow \mathcal{H}_{j-1}$ given by $V_{j-1, t} \eta:=\eta \circ F_{j-1, t}$. Due to the continuity of the map $\mathbb{R} \ni t \mapsto y_{t} \in \mathbb{T}^{\infty}$ and of the group operation, the family $\left\{V_{j-1, t}\right\}_{t \in \mathbb{R}}$ defines a strongly continuous unitary group in $\mathcal{H}_{j-1}$ with self-adjoint generator

$$
H_{j-1} \eta:=\mathrm{s}_{t \rightarrow 0} \lim _{t \rightarrow 0} i t^{-1}\left(V_{j-1, t}-1\right) \eta, \quad \eta \in \mathcal{D}\left(H_{j-1}\right):=\left\{\left.\eta \in \mathcal{H}_{j-1}\left|\lim _{t \rightarrow 0}\right| t\right|^{-1}\left\|\left(V_{j-1, t}-1\right) \eta\right\|<\infty\right\} .
$$

When dealing with differential operators on compact manifolds, one typically does the calculations on an appropriate core of the operators such as the set of $C^{\infty}$-functions. But here, the are no such functions on $\left(\mathbb{T}^{\infty}\right)^{j-1}$, since $\mathbb{T}^{\infty}$ is not a manifold $\left(\mathbb{T}^{\infty}\right.$ does not admit any differentiable structure modeled on locally convex spaces, see [4, Sec. 10.2] for details). So, we use instead the set $\mathcal{B}_{j-1}$ of Bruhat test functions on $\left(\mathbb{T}^{\infty}\right)^{j-1}$, whose definition is the following (see [3, Sec. 2.2] or [5] for details). Set $\mathbb{T}^{0}:=\{0\}$, and for each $n \in \mathbb{N}$ let $\pi_{n}:\left(\mathbb{T}^{\infty}\right)^{j-1} \rightarrow\left(\mathbb{T}^{n}\right)^{j-1}$ be the projection given by

$$
\pi_{n}\left(x_{1}, \ldots, x_{j-1}\right):= \begin{cases}(0, \ldots, 0) & \text { if } n=0 \\ \left(\left(x_{1}\right)_{1}, \ldots,\left(x_{1}\right)_{n}, \ldots,\left(x_{j-1}\right)_{1}, \ldots,\left(x_{j-1}\right)_{n}\right) & \text { if } n \geq 1\end{cases}
$$

Then,

$$
\mathcal{B}_{j-1}:=\bigcup_{n \in \mathbb{N}}\left\{\zeta \circ \pi_{n} \mid \zeta \in C^{\infty}\left(\left(\mathbb{T}^{n}\right)^{j-1}\right)\right\},
$$

that is, $\mathcal{B}_{j-1}$ is the set of all functions on $\left(\mathbb{T}^{\infty}\right)^{j-1}$ that are obtained by lifting to $\left(\mathbb{T}^{\infty}\right)^{j-1}$ any $C^{\infty}$-function on one of the Lie groups $\left(\mathbb{T}^{n}\right)^{j-1}$. The set $\mathcal{B}_{j-1}$ is dense in $\mathcal{H}_{j-1}$, it is left invariant by the group $\left\{V_{j-1, t}\right\}_{t \in \mathbb{R}}$, and satisfies the inclusion $\mathcal{B}_{j-1} \subset \mathcal{D}\left(H_{j-1}\right)$ (to show the latter, one has to use the $C^{1}$-assumption (3.1)). Therefore, it follows from Nelson's theorem [1, Prop. 5.3] that $H_{j-1}$ is essentially self-adjoint on $\mathcal{B}_{j-1}$.

Now, if the (multiplication operator valued) map $\mathbb{R} \ni t \mapsto \chi \circ \phi_{j-1} \circ F_{j-1, t} \in \mathscr{B}\left(\mathcal{H}_{j-1}\right)$ is strongly of class $C^{1}$, then $\chi \circ \phi_{j-1} \in C^{1}\left(H_{j-1}\right)$ since

$$
\chi \circ \phi_{j-1} \circ F_{j-1, t}=V_{j-1, t}\left(\chi \circ \phi_{j-1}\right) V_{j-1,-t}=e^{-i t H_{j-1}}\left(\chi \circ \phi_{j-1}\right) \mathrm{e}^{i t H_{j-1}} .
$$

Thus, the operator

$$
\begin{aligned}
g_{j, \chi}:=\left[H_{j-1},\left(\chi \circ \phi_{j-1}\right)\right]\left(\bar{\chi} \circ \phi_{j-1}\right) & \equiv \mathrm{s}-\left.\frac{\mathrm{d}}{\mathrm{d} t}\left(i \chi \circ \phi_{j-1} \circ F_{j-1, t}\right)\left(\bar{\chi} \circ \phi_{j-1}\right)\right|_{t=0} \\
& \equiv \mathrm{s}-\left.\frac{\mathrm{d}}{\mathrm{d} t} i \chi \circ\left(\phi_{j-1} \circ F_{j-1, t}-\phi_{j-1}\right)\right|_{t=0}
\end{aligned}
$$

is a bounded self-adjoint multiplication operator in $\mathcal{H}_{j-1}$.

Lemma 3.1. Take $j \in\{2, \ldots, d\}$ and $\chi \in \widehat{\mathbb{T}^{\infty}} \backslash\{1\}$, and assume that the map $\mathbb{R} \ni t \mapsto \chi \circ \phi_{j-1} \circ F_{j-1, t} \in$ $\mathscr{B}\left(\mathcal{H}_{j-1}\right)$ is strongly of class $C^{1}$. Then, $U_{j, \chi} \in C^{1}\left(H_{j-1}\right)$ with $\left[H_{j-1}, U_{j, \chi}\right]=g_{j, \chi} U_{j, \chi}$.

Proof. Take $\eta \in \mathscr{B}_{j-1}$. Then, the commutation of $V_{j-1, t}$ and $W_{j-1}$ and the differentiability assumption 
imply that

$$
\begin{aligned}
& \left\langle H_{j-1} \eta, U_{j, \chi} \eta\right\rangle_{\mathcal{H}_{j-1}}-\left\langle\eta, U_{j, \chi} H_{j-1} \eta\right\rangle_{\mathcal{H}_{j-1}} \\
& =\left.i \frac{\mathrm{d}}{\mathrm{d} t}\left\{-\left\langle V_{j-1, t} \eta,\left(\chi \circ \phi_{j-1}\right) W_{j-1} \eta\right\rangle_{\mathcal{H}_{j-1}}-\left\langle\eta,\left(\chi \circ \phi_{j-1}\right) W_{j-1} V_{j-1, t} \eta\right\rangle_{\mathcal{H}_{j-1}}\right\}\right|_{t=0} \\
& =\left.i \frac{\mathrm{d}}{\mathrm{d} t}\left\langle\eta,\left(\chi \circ \phi_{j-1} \circ F_{j-1, t}-\chi \circ \phi_{j-1}\right) W_{j-1} V_{j-1, t} \eta\right\rangle_{\mathcal{H}_{j-1}}\right|_{t=0} \\
& =\left.\frac{\mathrm{d}}{\mathrm{d} t}\left\langle\eta,\left\{\left(i \chi \circ \phi_{j-1} \circ F_{j-1, t}\right)\left(\bar{\chi} \circ \phi_{j-1}\right)-1\right\} U_{j, \chi} V_{j-1, t} \eta\right\rangle_{\mathcal{H}_{j-1}}\right|_{t=0} \\
& =\left.\frac{\mathrm{d}}{\mathrm{d} t}\left\langle\eta,\left\{i \chi \circ\left(\phi_{j-1} \circ F_{j-1, t}-\phi_{j-1}\right)-1\right\} U_{j, \chi} V_{j-1, t} \eta\right\rangle_{\mathcal{H}_{j-1}}\right|_{t=0} \\
& =\left\langle\eta, g_{j, \chi} U_{j, \chi} \eta\right\rangle_{\mathcal{H}_{j-1}},
\end{aligned}
$$

and thus the claim follows from the boundedness of $g_{j, \chi}$ and the density of $\mathscr{B}_{j-1}$ in $\mathcal{D}\left(\mathcal{H}_{j-1}\right)$.

Assumption 3.2. For each $j \in\{2, \ldots, d\}$, the map $\phi_{j-1} \in C\left(\left(\mathbb{T}^{\infty}\right)^{j-1} ; \mathbb{T}^{\infty}\right)$ satisfies $\phi_{j-1}=\xi_{j-1}+\eta_{j-1}$, where

(i) $\xi_{j-1} \in C\left(\left(\mathbb{T}^{\infty}\right)^{j-1} ; \mathbb{T}^{\infty}\right)$ is a group homomorphism such that

$$
\mathbb{T}^{\infty} \ni x_{j-1} \mapsto\left(\chi \circ \xi_{j-1}\right)\left(0, \ldots, 0+x_{j-1}\right) \in \mathbb{S}^{1}
$$

is nontrivial for each $\chi \in \widehat{\mathbb{T}^{\infty}} \backslash\{1\}$,

(ii) $\eta_{j-1} \in C\left(\left(\mathbb{T}^{\infty}\right)^{j-1} ; \mathbb{T}^{\infty}\right)$ is such that there exists $\widetilde{\eta}_{j-1} \in C\left(\left(\mathbb{T}^{\infty}\right)^{j-1} ; \mathbb{R}^{\infty}\right)$ with

$$
\eta_{j-1}\left(x_{1}, \ldots, x_{j-1}\right)=\left(\widetilde{\eta}_{j-1}\left(x_{1}, \ldots, x_{j-1}\right)\left(\bmod \mathbb{Z}^{\infty}\right)\right) \quad \text { for each }\left(x_{1}, \ldots, x_{j-1}\right) \in\left(\mathbb{T}^{\infty}\right)^{j-1},
$$

and with $\mathbb{R} \ni t \mapsto \widetilde{\eta}_{j-1, k} \circ F_{j-1, t} \in \mathscr{B}\left(\mathcal{H}_{j-1}\right)$ strongly of class $C^{1}$ for each $k \in \mathbb{N}_{\geq 1}$.

In the next theorem, we use the fact that the map

$$
\mathbb{R} \ni t \mapsto\left(\chi \circ \xi_{j-1}\right)\left(0, \ldots, 0+y_{t}\right) \in \mathbb{S}^{1}
$$

is a character on $\mathbb{R}$, and thus of class $C^{\infty}$. We also use the notation

$$
\xi_{j-1}^{(\chi)}:=\left.\frac{\mathrm{d}}{\mathrm{d} t}\left(\chi \circ \xi_{j-1}\right)\left(0, \ldots, 0+y_{t}\right)\right|_{t=0} \in i \mathbb{R} .
$$

Theorem 3.3 (Strong mixing and unique ergodicity). Suppose that Assumption 3.2 is satisfied. Then, $W_{d}$ is strongly mixing in $\mathcal{H}_{d} \ominus \mathcal{H}_{1}$ and $T_{d}$ is uniquely ergodic with respect to $\mu_{d}$.

These results of strong mixing and unique ergodicity are an extension to the case of infinitedimensional tori of results previously obtained in the case of one-dimensional tori (see [8, Thm. 2.1] for the unique ergodicity and [10, Rem. 1] for the strong mixing property). For instance, if the functions $\eta_{j-1}$ in Assumption 3.2 were to depend only on a finite number of variables, then the strong $C^{1}$ regularity condition on $\eta_{j-1}$ in Assumption 3.2(ii) would reduce to a uniform Lipschitz condition of $\eta_{j-1}$ along the flow $\left\{F_{j-1, t}\right\}_{t \in \mathbb{R}}$, as in the one-dimensional case treated by Furstenberg in [8, Thm. 2.1].

Proof. (i) Take $j \in\{2, \ldots, d\}, \chi \in \widehat{\mathbb{T}^{\infty}} \backslash\{1\}$ and $t \in \mathbb{R}$. Then, there exist $k_{\chi} \in \mathbb{N}_{\geq 1}$ and $n_{1}, \ldots, n_{k_{\chi}} \in \mathbb{Z}$ such that

$$
\chi\left(x_{j-1}\right)=\mathrm{e}^{2 \pi i \sum_{k=1}^{k \chi} n_{k} x_{j-1, k}},
$$


with $x_{j-1} \in \mathbb{T}^{\infty}$ and $x_{j-1, k} \in[0,1)$ the $k$-th cyclic component of $x_{j-1}$. Therefore, we infer from Assumption 3.2 that

$$
\begin{aligned}
\chi \circ\left(\phi_{j-1} \circ F_{j-1, t}-\phi_{j-1}\right) & =\chi \circ\left(\xi_{j-1} \circ F_{j-1, t}-\xi_{j-1}\right) \cdot \chi \circ\left(\eta_{j-1} \circ F_{j-1, t}-\eta_{j-1}\right) \\
& =\left(\chi \circ \xi_{j-1}\right)\left(0, \ldots, 0+y_{t}\right) \cdot e^{2 \pi i \sum_{k=1}^{k \chi} n_{k}\left(\widetilde{\eta}_{j-1} \circ F_{j-1, t}-\widetilde{\eta}_{j-1}\right)_{k}},
\end{aligned}
$$

and Lemma 3.1 implies that $U_{j, \chi} \in C^{1}\left(H_{j-1}\right)$ with $\left[H_{j-1}, U_{j, \chi}\right]=g_{j, \chi} U_{j, \chi}$ and

$$
\begin{aligned}
g_{j, \chi}=\mathrm{s}-\left.\frac{\mathrm{d}}{\mathrm{d} t} i \chi \circ\left(\phi_{j-1} \circ F_{j-1, t}-\phi_{j-1}\right)\right|_{t=0} & =i \xi_{j-1}^{(\chi)}-2 \pi \sum_{k=1}^{k_{\chi}} n_{k}\left(\mathrm{~s}-\left.\frac{\mathrm{d}}{\mathrm{d} t} \widetilde{\eta}_{j-1, k} \circ F_{j-1, t}\right|_{t=0}\right) \\
& =i \xi_{j-1}^{(\chi)}+2 \pi i \sum_{k=1}^{k_{\chi}} n_{k}\left(H_{j-1} \widetilde{\eta}_{j-1, k}\right) .
\end{aligned}
$$

(ii) We now proceed by induction on $d$ to prove the claims. For the case $d=2$, we take $\chi \in \widehat{\mathbb{T}^{\infty}} \backslash\{1\}$ and note that point (i) implies

$$
\begin{aligned}
& D_{2, \chi}:=\mathrm{s}_{N \rightarrow \infty} \lim \frac{1}{N} \sum_{\ell=0}^{N-1}\left(U_{2, \chi}\right)^{\ell}\left(\left[H_{1}, U_{2, \chi}\right]\left(U_{2, \chi}\right)^{-1}\right)\left(U_{2, \chi}\right)^{-\ell} \\
& =\underset{N \rightarrow \infty}{\mathrm{s}-\lim _{\boldsymbol{L}}} \frac{1}{N} \sum_{\ell=0}^{N-1} g_{2, \chi} \circ\left(T_{1}\right)^{\ell} \\
& =i \xi_{1}^{(\chi)}+2 \pi i \sum_{k=1}^{k_{\chi}} n_{k}\left(\operatorname{s}_{N \rightarrow \infty} \lim _{N} \frac{1}{N} \sum_{\ell=0}^{N-1}\left(H_{1} \widetilde{\eta}_{1, k}\right) \circ\left(T_{1}\right)^{\ell}\right) .
\end{aligned}
$$

Since $T_{1}$ is ergodic and $H_{1} \widetilde{\eta}_{1, k} \in \mathrm{L}^{\infty}\left(\mathbb{T}^{\infty}\right)$, it follows by Birkhoff's pointwise ergodic theorem and Lebesgue dominated convergence theorem that

$$
D_{2, \chi}=i \xi_{1}^{(\chi)}+2 \pi i \sum_{k=1}^{k_{\chi}} n_{k} \int_{\mathbb{T}^{\infty}} \mathrm{d} \mu_{1}\left(H_{1} \widetilde{\eta}_{1, k}\right)=i \xi_{1}^{(\chi)}+2 \pi i \sum_{k=1}^{k_{\chi}} n_{k} \int_{\mathbb{T}^{\infty}} \mathrm{d} \mu_{1}\left\langle 1, H_{1} \widetilde{\eta}_{1, k}\right\rangle_{\mathcal{H}_{1}}=i \xi_{1}^{(\chi)} .
$$

Now, since the character $\mathbb{T}^{\infty} \ni x_{j-1} \mapsto\left(\chi \circ \xi_{1}\right)\left(0, \ldots, 0+x_{j-1}\right) \in \mathbb{S}^{1}$ is nontrivial and the subgroup $\left\{y_{t}\right\}_{t \in \mathbb{R}}$ ergodic, we have $\xi_{1}^{(x)} \neq 0$ (see $\left[6\right.$, Thm. 4.1.1']). Thus, $D_{2, \chi} \neq 0$, and we deduce from Theorem 2.2(a) that $U_{2, x}$ is strongly mixing. Since this is true for each $\chi \in \overline{\mathbb{T}^{\infty}} \backslash\{1\}$, and since $U_{2, \chi}$ is unitarily

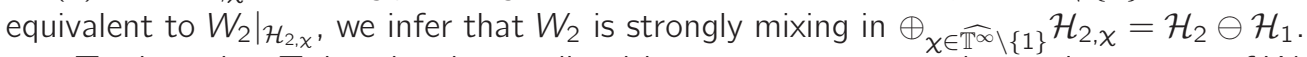

To show that $T_{2}$ is uniquely ergodic with respect to $\mu_{2}$, we take an eigenvector of $W_{2}$ with eigenvalue 1 , that is, a vector $\varphi \in \mathcal{H}_{2}$ such that $W_{2} \varphi=\varphi$. Since $W_{2}$ is strongly mixing in $\mathcal{H}_{2} \ominus \mathcal{H}_{1}, W_{2}$ has purely continuous spectrum in $\mathcal{H}_{2} \ominus \mathcal{H}_{1}$ (see Theorem 2.2(b)), and thus $\varphi=\eta \otimes 1$ for some $\eta \in \mathcal{H}_{1}$. So,

$$
W_{2} \varphi=\varphi \Longleftrightarrow W_{2}(\eta \otimes 1)=\eta \otimes 1 \Longleftrightarrow W_{1} \eta=\eta \text {. }
$$

and thus $\eta$ is an eigenvector of $W_{1}$ with eigenvalue 1 . It follows that $\eta$ is constant $\mu_{1}$-almost everywhere due to the ergodicity of $T_{1}$. Therefore, $\varphi$ is constant $\mu_{2}$-almost everywhere, and $T_{2}$ is ergodic. This implies that $T_{2}$ is uniquely ergodic because ergodicity implies unique ergodicity for transformations such as $T_{2}$ (see [6, Thm. 4.2.1]).

Now, assume the claims are true for $d-1 \geq 1$. Then, $W_{d-1}$ is strongly mixing in $\mathcal{H}_{d-1} \ominus \mathcal{H}_{1}$. Furthermore, a calculation as in the case $d=2$ shows that $W_{d}$ is strongly mixing in $\oplus_{\chi \in \widehat{\mathbb{T}} \backslash\{1\}} \mathcal{H}_{d, \chi}=$ $\mathcal{H}_{d} \ominus \mathcal{H}_{d-1}$. This implies that $W_{d}$ is strongly mixing in

$$
\left(\mathcal{H}_{d-1} \ominus \mathcal{H}_{1}\right) \oplus\left(\mathcal{H}_{d} \ominus \mathcal{H}_{d-1}\right)=\mathcal{H}_{d} \ominus \mathcal{H}_{1}
$$

This, together with the unique ergodicity of $T_{d-1}$, allows us to show that $T_{d}$ is uniquely ergodic as in the case $d=2$. 
We know from the proof of Theorem 3.3 that if Assumption 3.2 is satisfied, then $U_{j, x} \in C^{1}\left(H_{j-1}\right)$ with $\left[H_{j-1}, U_{j, \chi}\right]=g_{j, \chi} U_{j, \chi}$. So, it follows from [7, Sec. 4] that the operator

$$
A_{j, \chi}^{(N)} \eta:=\frac{1}{N} \sum_{\ell=0}^{N-1}\left(U_{j, \chi}\right)^{\ell} H_{j-1}\left(U_{j, \chi}\right)^{-\ell} \eta, \quad N \in \mathbb{N}_{\geq 1}, \eta \in \mathcal{D}\left(A_{j, \chi}^{(N)}\right):=\mathcal{D}\left(H_{j-1}\right),
$$

is self-adjoint, and that $U_{j, \chi} \in C^{1}\left(A_{j, \chi}^{(N)}\right)$ with

$$
\left[A_{j, \chi}^{(N)}, U_{j, \chi}\right]=g_{j, \chi}^{(N)} U_{j, \chi} \quad \text { and } \quad g_{j, \chi}^{(N)}:=\frac{1}{N} \sum_{\ell=0}^{N-1} g_{j, \chi} \circ\left(T_{j-1}\right)^{\ell} .
$$

Theorem 3.4 (Countable Lebesgue spectrum). Suppose that Assumption 3.2 is satisfied, and assume for each $j \in\{2, \ldots, d\}$ and $k \in \mathbb{N}_{\geq 1}$ that $H_{j-1} \widetilde{\eta}_{j-1, k} \in C\left(\left(\mathbb{T}^{\infty}\right)^{j-1}\right)$ and that

$$
\int_{0}^{1} \frac{\mathrm{d} t}{t}\left\|\left(H_{j-1} \widetilde{\eta}_{j-1, k}\right) \circ F_{j-1, t}-\left(H_{j-1} \widetilde{\eta}_{j-1, k}\right)\right\|_{L^{\infty}\left((\mathbb{T} \infty)^{j-1}\right)}<\infty .
$$

Then, $W_{d}$ has countable Lebesgue spectrum in $\mathcal{H}_{d} \ominus \mathcal{H}_{1}$.

Proof. Take $j \in\{2, \ldots, d\}$ and $\chi \in \widehat{\mathbb{T}^{\infty}} \backslash\{1\}$. Then, we know from Lemma 3.1 that $U_{j, x} \in C^{1}\left(H_{j-1}\right)$ with $\left[H_{j-1}, U_{j, \chi}\right]=g_{j, \chi} U_{j, \chi}$. Furthermore, (3.2) and (3.3) imply that

$$
\begin{aligned}
& \int_{0}^{1} \frac{\mathrm{d} t}{t}\left\|\mathrm{e}^{-i t H_{j-1}} g_{j, \chi} \mathrm{e}^{i t H_{j-1}}-g_{j, \chi}\right\|_{\mathscr{B}\left(\mathcal{H}_{j-1}\right)} \\
& =\int_{0}^{1} \frac{\mathrm{d} t}{t}\left\|g_{j, \chi} \circ F_{j-1, t}-g_{j, \chi}\right\|_{L^{\infty}\left(\left(\mathbb{T}^{\infty}\right)^{j-1}\right)} \\
& \leq 2 \pi \sum_{k=1}^{k_{\chi}}\left|n_{k}\right| \int_{0}^{1} \frac{\mathrm{d} t}{t}\left\|\left(H_{j-1} \widetilde{n}_{j-1, k}\right) \circ F_{j-1, t}-\left(H_{j-1} \widetilde{\eta}_{j-1, k}\right)\right\|_{L^{\infty}\left(\left(\mathbb{T}^{\infty}\right)^{j-1}\right)} \\
& <\infty .
\end{aligned}
$$

So, we obtain that $U_{j, x} \in C^{1+0}\left(H_{j-1}\right)$ with $\left[H_{j-1}, U_{j, x}\right]=g_{j, x} U_{j, x}$, and thus deduce from [7, Sec. 4] that $U_{j, \chi} \in C^{1+0}\left(A_{j, \chi}^{(N)}\right)$ with $\left[A_{j, \chi}^{(N)}, U_{j, \chi}\right]=g_{j, \chi}^{(N)} U_{j, \chi}$. Now, $H_{j-1} \widetilde{\eta}_{j-1, k} \in C\left(\left(\mathbb{T}^{\infty}\right)^{j-1}\right)$ and $T_{j-1}$ is uniquely ergodic with respect to $\mu_{j-1}$ due to Theorem 3.3. Thus, we infer from (3.2) that

$$
\begin{aligned}
\lim _{N \rightarrow \infty} g_{j, \chi}^{(N)} & =i \xi_{j-1}^{(\chi)}+2 \pi i \sum_{k=1}^{k_{\chi}} n_{k} \frac{1}{N} \sum_{\ell=0}^{N-1}\left(H_{j-1} \widetilde{\eta}_{j-1, k}\right) \circ\left(T_{j-1}\right)^{\ell} \\
& =i \xi_{j-1}^{(\chi)}+2 \pi i \sum_{k=1}^{k_{\chi}} n_{k} \int_{\left(\mathbb{T}^{\infty}\right)^{j-1}} \mathrm{~d} \mu_{j-1}\left\langle 1, H_{j-1} \widetilde{\eta}_{j-1, k}\right\rangle_{\mathcal{H}_{j-1}} \\
& =i \xi_{j-1}^{(\chi)}
\end{aligned}
$$

uniformly on $\left(\mathbb{T}^{\infty}\right)^{j-1}$. Since $\xi_{j-1}^{(\chi)} \neq 0$ by the proof of Theorem 3.3, one has $\left|g_{j, \chi}^{(N)}\right|>0$ if $N$ is large enough. So, $\left|g_{j, \chi}^{(N)}\right| \geq a$ with $a:=\inf _{x \in\left(\mathbb{T}^{\infty}\right)^{j-1}}\left|g_{j, \chi}^{(N)}(x)\right|>0$, and $U_{j, \chi}$ satisfies the following strict Mourre estimate on $\mathbb{S}^{1}$ :

$$
\left(U_{j, \chi}\right)^{*}\left[\operatorname{sgn}\left(g_{j, \chi}^{(N)}\right) A_{j, \chi}^{(N)}, U_{j, \chi}\right]=\left(U_{j, \chi}\right)^{*}\left|g_{j, \chi}^{(N)}\right| U_{j, \chi} \geq a
$$

Therefore, all the assumptions of Theorem 2.1 are satisfied, and thus $U_{j, x}$ has purely absolutely continuous spectrum. Since this occurs for each $j \in\{2, \ldots, d\}$ and $\chi \in \widehat{\mathbb{T}^{\infty}} \backslash\{1\}$, and since $U_{j, x}$ is unitarily equivalent to $\left.W_{d}\right|_{\mathcal{H}_{j, x}}$, one infers that $W_{d}$ has purely absolutely continuous spectrum in $\bigoplus_{j \in\{2, \ldots, d\}, \chi \in \mathbb{\mathbb { T }}_{\infty} \backslash\{1\}} \mathcal{H}_{j, \chi}=$ $\mathcal{H}_{d} \ominus \mathcal{H}_{1}$. Finally, the fact that $W_{d}$ has has countable Lebesgue spectrum in $\mathcal{H}_{d} \ominus \mathcal{H}_{1}$ can be shown in a similar way as in [10, Lemmas $3 \& 4$ ]. 
The result of Theorem 3.4 is an extension to the case of infinite-dimensional tori of results previously obtained in the case of one-dimensional tori (see [10, Cor. 3] or [15, Thm. 5.3]).

\section{References}

[1] W. O. Amrein. Hilbert space methods in quantum mechanics. Fundamental Sciences. EPFL Press, Lausanne, 2009.

[2] W. O. Amrein, A. Boutet de Monvel, and V. Georgescu. Co-groups, commutator methods and spectral theory of N-body Hamiltonians, volume 135 of Progress in Mathematics. Birkhäuser Verlag, Basel, 1996.

[3] A. Bendikov and L. Saloff-Coste. On the sample paths of Brownian motions on compact infinite dimensional groups. Ann. Probab., 31(3):1464-1493, 2003.

[4] V. I. Bogachev. Differentiable measures and the Malliavin calculus. J. Math. Sci. (New York), 87(4):3577-3731, 1997. Analysis, 9.

[5] F. Bruhat. Distributions sur un groupe localement compact et applications à l'étude des représentations des groupes §-adiques. Bull. Soc. Math. France, 89:43-75, 1961.

[6] I. P. Cornfeld, S. V. Fomin, and Y. G. Sină. Ergodic theory, volume 245 of Grundlehren der Mathematischen Wissenschaften [Fundamental Principles of Mathematical Sciences]. Springer-Verlag, New York, 1982. Translated from the Russian by A. B. SosinskiI.

[7] C. Fernández, S. Richard, and R. Tiedra de Aldecoa. Commutator methods for unitary operators. J. Spectr. Theory, 3(3):271-292, 2013.

[8] H. Furstenberg. Strict ergodicity and transformation of the torus. Amer. J. Math., 83:573-601, 1961.

[9] G. Greschonig. Nilpotent extensions of Furstenberg transformations. Israel J. Math., 183:381-397, 2011.

[10] A. Iwanik, M. Lemańczyk, and D. Rudolph. Absolutely continuous cocycles over irrational rotations. Israel J. Math., 83(1-2):73-95, 1993.

[11] R. Ji. ON THE CROSSED PRODUCT C*-ALGEBRAS ASSOCIATED WITH FURSTENBERG TRANSFORMATIONS ON TORI. ProQuest LLC, Ann Arbor, MI, 1986. Thesis (Ph.D.)-State University of New York at Stony Brook.

[12] H. Osaka and N. C. Phillips. Furstenberg transformations on irrational rotation algebras. Ergodic Theory Dynam. Systems, 26(5):1623-1651, 2006.

[13] K. Reihani. K-theory of Furstenberg transformation group $C^{*}$-algebras. Canad. J. Math., 65(6):1287-1319, 2013.

[14] R. Tiedra de Aldecoa. Commutator criteria for strong mixing. to appear in Ergodic Theory Dynam. Systems, preprint on http://arxiv.org/abs/1406.5777.

[15] R. Tiedra de Aldecoa. Commutator methods for the spectral analysis of uniquely ergodic dynamical systems. Ergodic Theory and Dynamical Systems, FirstView:1-24, 102014. 